\title{
Step-Wise Group Screening Designs with Unequal A-Priori Probabilities and Errors in Observations
}

\author{
M.M. Manene
}

\author{
Department of Mathematics, University of Nairobi, P.O. Box 30197, Nairobi, Kenya, \\ Email: mathuon@iconnect.co.ke.
}

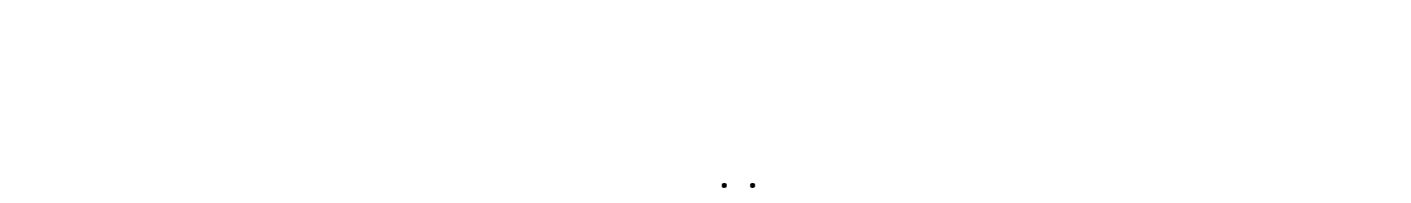

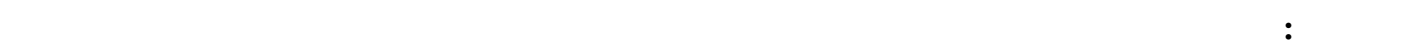

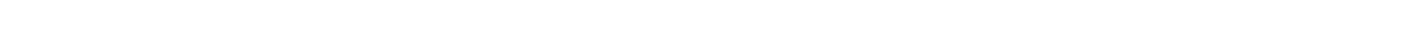

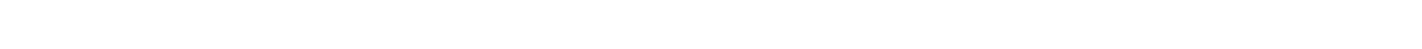

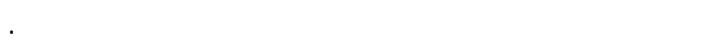

\begin{abstract}
The performance of step-wise group screening with unequal a-priori probabilities in terms of the expected number of runs and the expected maximum number of incorrect decisions is considered. A method of obtaining optimal step-wise designs with unequal a-priori probabilities is presented for the case in which the direction of each defective factor is assumed to be known a priori and observations are subject to error. An appropriate cost function is introduced and the value of the group size which minimizes the expected total cost is obtained.
\end{abstract}

KEYWORDS: Step-Wise, Designs, Group-Factors, Initial Step, Subsequent Steps, Expected Number of Runs, Incorrect Decisions, Cost Function.

\section{Introduction}

$\mathbf{T}$ here are investigations where a large number of factors needs to be examined. In such a situation we have to run an experiment to identify the influential factors. The group screening procedure aims at reducing the size of the experiment, thus conserving resources.

The method of group testing was first introduced by Dorfman (1943), who proposed that instead of testing each blood sample individually for the presence of a rare disease, blood samples be pooled and analysed together.

Watson (1961) considered two stage group screening designs with and without errors in observations and with equal prior probabilities. In the same paper, he laid down the device of using different group sizes when prior probabilities differ. Li (1962) and Patel (1962) generalized Watson's method to more than two stages. Both these authors considered multistage groupscreening designs with equal prior probabilities and without errors in observations. Ottieno and Patel (1984) extended the idea of two stage group screening with unequal prior probabilities to include situations when no prior information is available so that no natural partitioning can be assumed. Odhiambo and Patel (1986) generalized this approach to multi-stage designs.

The group testing procedure first considered by Sterrett (1957) has been extended by Manene (1985), Patel and Manene (1987), Odhiambo and Manene (1987) and Manene (1997) in what they have called step-wise group-screening designs and they have approached the problem from the 


\section{M.M. MANENE}

point of view of designs of experiments. They have considered the cases when all factors are defective with equal prior probabilities.

In this paper, we shall extend group screening designs considered by Odhiambo and Manene (1987) to the case when factors are defective with unequal prior probabilities.

\section{Assumptions and design structure}

We shall assume that there is a single response variable of interest $y$, which is related to a set of $f$ factors through the first order linear regression model

$$
y_{u}=\beta_{0}+\sum_{j=1}^{f} \beta_{j} x_{u j}+\varepsilon_{u}
$$

where $y_{u}$ is the $u^{\text {th }}$ response, $\beta_{0}$ is a constant term common to every response; $\beta_{j}(j \geq 1)$ is the linear effect of the $j^{\text {th }}$ factor, $x_{u j}= \pm 1$ is the level of the $j^{\text {th }}$ factor in the $u^{\text {th }}$ run, $\varepsilon_{u}$ is the $u^{\text {th }}$ error term. Further, we shall assume that it is possible to partition the $f$ factors into a fixed number $g$ of groups, such that the $i^{\text {th }}$ group contains $k_{i}$ factors. The factors will be partitioned into groups of unequal sizes by selecting a set of numbers $\left\{p_{1} \leq p_{2} \leq \ldots . \leq p_{g} ; \mathrm{o}<p_{i}<1\right\}$ and identifying $\mathrm{p}_{\mathrm{i}}$ as the probability that a factor belonging to the $i^{\text {th }}$ group is defective. Thus $p_{i}{ }^{\prime} s$ and $k_{i}{ }^{\prime} s$ will be variables. This is a generalization of natural partitioning, when $p_{i}{ }^{\prime} s$ are actual probabilities. Thus, in addition to model (2.1), we shall make the following assumptions;

(i) The total number of factors, $f$, can be divided into a fixed number ' $g$ ' of group-factors in the initial step such that $f=\sum_{i=1}^{g} k_{i}$ where $k_{i}$ is the number of factors in the $i^{\text {th }}$ group factor.

(ii) The errors are independent normal with means zero and variance $\sigma^{2}$ (known).

(iii) All the factors in the $i^{\text {th }}$ group-factor have independently the same probability $p_{i}$ (variable) of being defective.

(iv) A defective factor within the $i^{\text {th }}$ group-factor has a positive effect $\Delta_{i}$.

The approach here is rather similar to the use of diffuse prior distributions in Bayesian inference.

The step-wise group-screening experiment is performed in steps as follows: in the initial step, the $f$ factors are divided into $g$ groups such that the $i^{\text {th }}$ group contains $k_{i}$ factors $(i=1,2, \ldots, g)$. These groups are called group factors. These group-factors are then tested for significance. Those that are declared non-defective are set aside. In step two, we start with any group- factor that is declared defective in the initial step and examine the factors within it one by one till a factor is declared defective. We set aside factors which are declared non-defective, keeping the factor declared defective separate. The remaining factors are then tested in a group. This is done for all group-factors declared defective in the initial step. The test procedure carried out in the initial step and in step two is repeated in subsequent steps successively till the analysis terminates with a group-factor declared non-defective or with a group-factor of size one.

In testing the significance of the group-factors in the initial step, we shall use the orthogonal main effects plans of the type given by Placket and Burman (1946). For testing the significance of individual factors and group-factors in the subsequent steps we shall use non orthogonal designs to simplify computations. 


\section{STEP-WISE GROUP SCREENIN DESIGNS}

\section{Expected Number of Runs}

Suppose that $f$ factors are divided into a fixed number ' $g$ ' of group-factors in the initial step such that the $i^{\text {th }}$ group-factor is of size $k_{i}\left(\sum_{i=1}^{g} k_{i}=f\right)$. The ' $g$ ' group-factors are tested in

$$
\begin{aligned}
R(g) & =g+4-g(\bmod 4) \\
& =g+h(h=1,2,3,4)
\end{aligned}
$$

runs. In the subsequent steps, factors within the defective group-factors are tested as explained earlier.

Let $\hat{G}_{i}$ be the estimate of the main effect of the $i^{\text {th }}$ group-factor in the initial step, with $\delta_{i}$ effective factors each with effect $\Delta_{i}>0 ;\left(\delta_{i}=1,2, \ldots, k_{i}\right)$. Then $E\left(G_{i}\right)=\delta_{i} \Delta_{i}$ and $\operatorname{Var}$ $\left(\hat{G}_{i}\right)=\sigma^{2} / g+h$.

Define a random variable $W_{i}$ by

$$
W_{i}=\hat{G}_{i} / \sigma \sqrt{g+h}
$$

Then

$$
E\left(W_{i}\right)=\delta_{i} u_{i} \text { and } \operatorname{Var}\left(W_{i}\right)=1, \text { where } u_{i}=\Delta_{i} / \sigma \sqrt{g+h}
$$

Consider the hypothesis $H_{0}: \delta_{i} u_{i}=0$ alternative $u_{i} \delta_{i}=0$. Let $\alpha_{\mathrm{Ii}}$ be the level of significance for testing the $\mathrm{i}^{\text {th }}$ group-factor in the initial step and denote by $\Pi_{\mathrm{Ii}}\left(\delta_{i} u_{i}, \alpha_{\mathrm{Ii}}\right)$ the power function of the test. Then

$$
\Pi_{\mathrm{Ii}}\left(\delta_{i} u_{i}, \alpha_{\mathrm{Ii}}\right)=1-\phi\left(Z\left(\alpha_{\mathrm{Ii}}\right)-\delta_{i} u_{i}\right)
$$

Where $\phi($.$) denotes the standard normal distribution function and Z\left(\alpha_{\text {Ii }}\right)$ satisfies

$$
\alpha_{\mathrm{Ii}}=1-\phi\left(Z\left(\alpha_{\mathrm{Ii}}\right)\right)
$$

Thus if $\delta_{i}=0$ for $\mu_{\mathrm{i}}=0$, then $\Pi_{\mathrm{Ii}}\left(0, \alpha_{\mathrm{Ii}}\right)=\alpha_{\mathrm{Ii}}$ and if $\delta_{i} \neq 0$ and $\Delta_{1} / \sigma$ is large, then $\Pi_{\mathrm{Ii}}\left(\delta_{i} u_{i}, \alpha_{\mathrm{Ii}}\right)$ tends to 1 .

Let $\Pi_{\mathrm{Ii}}^{*}$ denote the probability that the $i^{\text {th }}$ group factor is declared defective in the initial step. Then

$$
\Pi_{\mathrm{Ii}}^{*}=\sum_{\delta_{i}=0}^{k i}\left(\begin{array}{l}
k_{i} \\
\delta_{i}
\end{array}\right) p_{i}^{\delta_{i}}\left(1-p_{i}\right)^{k_{i}-\delta_{i}} \Pi_{\mathrm{Ii}}\left(\delta_{i}, u_{i}, \alpha_{\mathrm{Ii}}\right)
$$

where $p_{i}$ is the probability that a factor in the $i^{\text {th }}$ group-factor in the initial step is defective. Define a random variable $U_{i}$ such that

$$
U_{i}= \begin{cases}1 & \text { with probability } \Pi_{\mathrm{Ii}}^{*} \\ 0 & \text { otherwise } \quad(i=1,2, \ldots g)\end{cases}
$$

Then

$$
E\left(U_{i}\right)=\Pi_{\mathrm{Ii}}^{*}
$$




\section{M.M. MANENE}

In the subsequent steps, we shall use non-orthogonal designs. Let $p_{i}{ }_{i}$ be the probability that a factor chosen at random from the $i^{\text {th }}$ group factor containing $\delta_{i}$ defective factors that has been declared defective in the initial step is defective.

Then

$$
p^{\prime}{ }_{i}=p_{i} / \Pi_{I i}^{*} \sum_{\delta_{i}=1}^{k_{i}}\left(\begin{array}{c}
k_{i}-1 \\
\delta_{i}-1
\end{array}\right) p_{i}^{\delta_{i}-1} q_{i}^{k_{i}-\delta_{i}} \Pi_{I i}\left(\delta_{i} \mu_{i}, \alpha_{I i}\right)=p_{i} \Pi_{I i}^{+} / \Pi_{I i}^{*}
$$

where

$$
\Pi_{I i}^{+}=\sum_{\delta_{i}=1}^{k_{i}}\left(\begin{array}{c}
k_{i}-1 \\
\delta_{i}-1
\end{array}\right) p_{i}^{\delta_{i}-1}\left(1-p_{i}\right)^{k_{i}-\delta_{i}} \Pi_{\mathrm{Ii}}\left(\delta_{i} \mu_{i}, \alpha_{I i}\right)
$$

Let $\alpha_{s i}$ be the probability of declaring a non-defective factor from the $i^{\text {th }}$ group-factor in the initial step as defective and $\gamma_{s i}$ be the probability of declaring a defective factor as defective in the subsequent steps. Further let $\bar{\beta}_{i}^{+}$be the probability that a factor chosen at random from the $i^{\text {th }}$ group-factor in the initial step is declared defective in the subsequent steps if the group-factor was declared defective in the initial step.

Then

$$
\begin{aligned}
\bar{\beta}_{i}^{+} & =\gamma_{s i} p_{i}{ }_{i}+\alpha_{s i}\left(1-p^{\prime}{ }_{i}\right) \\
& =\left[p_{i}\left(\gamma_{s i}-\alpha_{s i}\right) \Pi_{\mathrm{Ii}}^{+}+\Pi_{\mathrm{Ii}}^{+} \alpha_{s i}\right] / \Pi_{I i}^{*}=\bar{\beta}_{i}^{*} / \Pi_{I i}^{*}
\end{aligned}
$$

where

$$
\bar{\beta}_{i}^{*}=\left[p_{i}\left(\gamma_{s i}-\alpha_{s i}\right) \Pi_{\mathrm{Ii}}^{+}+\Pi_{\mathrm{Ii}}^{*} \alpha_{s i}\right]
$$

Let $\alpha_{s i}^{*}$ be the probability of declaring a non-defective factor from the $i^{\text {th }}$ group-factor in the initial step group-factors is declared defective at any step but on testing individual factors within it, no factor is declared defective due to errors in observations. Obviously $\alpha_{s i}^{*}$ will take different values at different steps. However for simplicity in algebra, we shall assume $\alpha_{s i}^{*}$ to be of uniform value, say $\alpha_{i}{ }^{*}$. Denote by $p_{k_{j}}{ }^{*}(j)$ the probability that exactly $j$ factors from the $i^{\text {th }}$ group-factor in the initial step that has been declared defective in the subsequent steps.

Then

$$
p_{k_{i}}^{*}(j)=\left\{\begin{array}{l}
1-\frac{1}{\Pi_{I i}^{*}}\left[\left(1-\left(1-\bar{\beta}_{i}^{*}\right)^{k_{i}}\right)\right] ; j=0 \\
\frac{1}{\Pi_{I i}^{*}}\left(\begin{array}{c}
k_{i} \\
j
\end{array}\right) \bar{\beta}_{i}^{* j}\left(1-\bar{\beta}_{i}^{*}\right)^{k_{i}-j} ; \quad\left(j=1,2, \ldots \ldots ., k_{i}\right)
\end{array}\right.
$$

Let $E_{k_{i}}^{*}(R)$ be the expected number of runs required to declare exactly $j$ factors defective from the $i^{t h}$ initial step group-factor which has been declared defective. Then following Odhiambo and Manene (1987), 


\section{STEP-WISE GROUP SCREENIN DESIGNS}

$$
E_{k_{i}}^{*}\left(R_{j}\right)=\left\{\begin{array}{l}
k_{i} \quad \text { for } \quad j=0 \\
\frac{j k_{i}}{j+1}+j+\frac{j}{j+1}-\frac{j\left(k_{i}+j-2\right)}{k_{i}\left(k_{i}-1\right)}+\alpha_{i}^{*}\left\{\frac{k_{i}}{j+1}-\frac{j}{j+1}-\frac{j}{k_{i}-1}+\frac{j^{2}}{k_{i}\left(k_{i}-1\right)}\right.
\end{array}\right\}
$$

where $\alpha_{i}^{*}$ is as already defined and $\xi_{i}=0$ if $\alpha_{i}^{*}=0$ and 1 otherwise.

Let $R_{s i}$ be the number of runs required to analyse the $i^{\text {th }}$ group-factor once it has been declared defective in the initial step. Then

$$
\begin{aligned}
E\left(R_{s i}\right) & =\sum_{j=1}^{k_{i}} E_{k_{i}}^{*}\left(R_{j}\right) p_{k_{i}}^{*}(j) \\
& =k_{i}-\frac{k_{i}}{\Pi_{I i}}\left[1-\left(1-\bar{\beta}_{i}^{*}\right)^{k_{i}}\right]-\frac{1}{\Pi_{I i}^{*}}\left[\left(2-\xi_{i}\right) \bar{\beta}_{i}^{*}+\xi_{i} \bar{\beta}_{i}^{* 2}\right] \\
& +\frac{1}{\Pi_{I i}}\left[k_{i}+1+k_{i} \bar{\beta}_{i}^{*}-\frac{1}{\bar{\beta}_{i}^{*}}\left\{1-\left(1-\bar{\beta}_{i}^{*}\right)^{k_{i}+1}\right\}\right] \\
& +\frac{\alpha_{i}^{*}}{\Pi_{I i}^{*}}\left[\frac{1}{\bar{\beta}_{i}^{*}}\left\{1-\left(1-\bar{\beta}_{i}^{*}\right)^{k_{i}+1}-k_{i} \bar{\beta}_{i}^{*}\left(1-\bar{\beta}_{i}^{*}\right)^{k_{i}}-1-\bar{\beta}_{i}^{*}+\bar{\beta}_{i}^{* 2}\right]\right.
\end{aligned}
$$

Using (3.13) and (3.14). If $R_{s}$ is the number of runs required to analyse all the group factors declared defective in the initial step, then

$$
R_{s}=\sum_{i=1}^{g} U_{i} E\left(R_{s i}\right)
$$

Theorem 3.1: The expected total number of runs in a step-wise group screening design with $g$ (fixed) group-factors in the initial step such that the $i^{\text {th }}$ group-factor is of size $k_{i}(i=1,2, \ldots, g)$ is given by

$$
\begin{aligned}
E(R) & =h+f+2 g-\sum_{i=1}^{g} \frac{1}{\bar{\beta}_{i}^{*}}\left(1-\alpha_{i}^{*}\right)\left\{1-\left(1-\bar{\beta}_{i}^{*}\right)^{k_{i}+1}\right\} \\
& +\sum_{i=1}^{g} k_{i} \bar{\beta}_{i}^{*}\left\{1-\frac{2-\xi_{i}}{k_{i}}-\frac{\alpha_{i}^{*}}{k_{i}}\right\}+\sum_{i=1}^{g} k_{i} \Pi_{I i}^{*} \\
& -\sum_{i=1}^{g} k_{i}\left[1-\left(1-\bar{\beta}_{i}^{*}\right)^{k_{i}}\right]-\sum_{i=1}^{g} \alpha_{i}^{*}+\sum_{i=1}^{g}\left(\alpha_{i}^{*}-\xi_{i}\right) \bar{\beta}_{i}^{* 2} \\
& -\sum_{i=1}^{g} k_{i} \alpha_{i}^{*}\left(1-\bar{\beta}_{i}^{*}\right)^{k_{i}}
\end{aligned}
$$

Where $\bar{\beta}_{i}^{*}, \alpha_{i}^{*}$ and $\xi_{i}$ are as defined earlier.

Proof: In the initial step we require $R_{1}=g+h$ runs $(h=1,2,3,4)$. The number of runs 
required in the subsequent steps is

$$
R_{s}=\sum_{i=1}^{g} U_{i} E\left(R_{s i}\right)
$$

with

$$
E\left(R_{s}\right)=E\left\{\sum_{i=1}^{g} U_{i} E\left(R_{s i}\right)\right\}
$$

The theorem then follows on using (3.8) and (3.15) in (3.17), simplifying and noting that

$$
E(R)=R_{I}+E\left(R_{s}\right) \text {. }
$$

Corollary 3.1: For large values of $\frac{\Delta_{i}{ }^{\prime} s}{\sigma}$ and small values of $p_{i}{ }^{\prime} s$, the expected number of runs in a step-wise group screening design with $g$ group-factors in the initial step, the $i^{\text {th }}$ group- factor being of size $k_{i}(i=1,2, \ldots \ldots, g)$ is approximately equal to

$$
\begin{aligned}
& (g+h)+\sum_{i=1}^{\mathrm{g}}\left(1-\alpha_{s i}\right) k_{i} p_{i}\left\{1-\frac{2-\xi_{i}}{k_{i}}-k_{i}+\frac{1}{2}\left(1-\alpha_{i}^{*}\right)\left(k_{i}+1\right)\right\} \\
& +\sum_{i=1}^{\mathrm{g}} \alpha_{I i} k_{i}+\sum_{i=1}^{\mathrm{g}} k_{1}^{2}\left(1-\alpha_{I i}\right) p_{i}
\end{aligned}
$$

Proof: If $\frac{\Delta_{i}{ }^{\prime} s}{\sigma}$ are large, then

$$
\begin{gathered}
\Pi_{I i}^{*} \approx 1-\left(1-\alpha_{I i}\right) q_{i}^{k_{i}}, \Pi_{I i} \approx 1 \\
\gamma_{s i} \approx 1 \text { and } \beta_{i}^{*} \approx\left(1-\alpha_{s i}\right) p_{i}+\alpha_{s i}\left\lfloor 1-\left(1-\alpha_{I i}\right) q_{i}^{k_{i}}\right\rfloor
\end{gathered}
$$

Further if $p_{i}{ }^{\prime} s$ are small, then

$$
1-\left(1-\alpha_{I i}\right) q_{i}^{k_{i}} \approx\left(1-\alpha_{I i}\right) k_{i} p_{i}+\alpha_{I i}
$$

up to order $p_{i}$. The corollary then follows on using these approximations in the expression for $E(\mathrm{R})$ given in theorem 3.1 .

\section{Calculation of the expected number of incorrect decisions}

We shall consider the same cases of incorrect decisions as were considered by Odhiambo and Manene (1987) i.e.

(i) declaring defective factors as non-defective in the initial step

(ii) declaring defective factors as non-defective in subsequent steps

(iii) declaring non-defective factors as defective in the subsequent steps

Let $E_{k_{i}}(j)$ denote the expected number of factors declared defective from the $i^{\text {th }}$ group-factor that is declared defective in the initial step. Then

$$
E_{k_{i}}(j) \frac{1}{\Pi_{I i}^{*}} k_{i} \bar{\beta}_{i}^{+}=k_{i} \bar{\beta}_{i}^{+}
$$

where

$$
\bar{\beta}_{i}^{+}=\bar{\beta}_{i}^{*} / \Pi_{I i}^{*}
$$

Let $\underline{d}$ be the number of factors declared defective in the subsequent steps. Then 


\section{STEP-WISE GROUP SCREENIN DESIGNS}

$$
\underline{d}=\sum_{i=1}^{\mathrm{g}} k_{i} \bar{\beta}_{i}^{+} U_{i}
$$

where $U_{i}$ is as defined in (3.7).

Let $p_{i}{ }^{(0)}$ be the probability that a factor chosen at random from the $i^{\text {th }}$ initial step group- factor declared non-defective, is defective. Then

$$
p_{i}^{(0)}=p_{i}\left(1-\Pi_{I i}^{+}\right) /\left(1-\Pi_{I i}^{*}\right)
$$

Let $p_{i}^{+}$be the probability that a factor from the $i^{\text {th }}$ group factor is non-defective given that it is declared defective. Then

$$
p_{i}^{+}=\alpha_{s i}\left(1-p_{i}^{\prime}\right) / \bar{\beta}_{i}^{+}
$$

Theorem 4.1 : Let $M_{R}$ be the number of defective factors declared defective in a step-wise group screening design with $g$ initial group-factors, the factors in the $i^{\text {th }}$ group-factor of size $k_{i}$ being defective with a priori probability $p_{i}(i=1,2, \ldots ., g)$. Then

$$
E\left(M_{R}\right)=\sum_{\mathrm{i}=1}^{\mathrm{g}} k_{i} p_{i} \Pi_{I i}^{+} \gamma_{s i}
$$

Proof: The expected total number of factors declared defective in the subsequent steps is given by

$$
E(\underline{d})=\sum_{i=1}^{\mathrm{g}} k_{i} \bar{\beta}_{i}^{+} E\left(U_{i}\right)=\sum_{i=1}^{\mathrm{g}} k_{i} \bar{\beta}_{i}^{+} \Pi_{I i}^{*}
$$

The probability that a factor which is declared defective from the $i^{\text {th }}$ group-factor, is defective is given by $1-p_{i}^{+}$.

Therefore

$$
\begin{aligned}
E\left(M_{R}\right) & =\sum_{i=1}^{\mathrm{g}} k_{i} \bar{\beta}_{i}^{+}\left(1-p_{i}^{+}\right) E\left(U_{i}\right) \\
& =\sum_{i=1}^{\mathrm{g}} k_{i}\left[\bar{\beta}_{i}^{+}-\alpha_{s i}\left(1-P^{\prime}\right)\right] \Pi_{I i}^{*} \\
& =\sum_{i=1}^{\mathrm{g}} k_{i} p_{i} \Pi_{I i}^{*} \gamma_{s i}
\end{aligned}
$$

as required.

Let $I_{I}$ denote the expected number of defective factors declared non-defective in the initial step. Then

$$
\begin{aligned}
I_{I} & =E\left[\sum_{i=1}^{\mathrm{g}}\left(1-U_{i}\right) k_{i} p_{i}^{(0)}\right] \\
& =\sum_{i=1}^{\mathrm{g}} k_{i} p_{i}\left(1-\Pi_{I i}^{+}\right)
\end{aligned}
$$

Theorem 4.2 : In a step-wise group screening design with errors in observations and unequal apriori probabilities, the expected number of defective factors declared non-defective in the subsequent steps is given by

$$
I_{s}=\sum_{i=1}^{\mathrm{g}} k_{i} p_{i} \Pi_{I i}\left(1-\gamma_{s i}\right)
$$




\section{M.M. MANENE}

Proof: The expected total number of defective factors in all the $g$ group-factors in the initial step is equal to $\sum_{i=1}^{g} k_{i} p_{i}$. Therefore

$$
\begin{aligned}
I_{s} & =E\left[\sum_{i=1}^{g} k_{i} p_{i}-\sum_{i=1}^{g}\left(1-U_{i}\right) k_{i} p_{i}^{(0)}-M_{R}\right] \\
& =\sum_{i=1}^{g} k_{i} p_{i} \Pi_{I i}^{+}\left(1-\gamma_{s i}\right)
\end{aligned}
$$

This completes the proof.

Theorem 4.3 : Let $M_{u}$ be the number of non-defective factors declared defective in the subsequent steps. Then

$$
E\left(M_{u}\right)=\sum_{i=1}^{\mathrm{g}} k_{i} \alpha_{s i}\left(\Pi_{I i}^{*}-p_{i} \Pi_{I i}^{+}\right)
$$

Proof: The total number of factors declared defective in the subsequent steps is $\sum_{i=1}^{\mathrm{g}} k_{i} \bar{\beta}_{i}^{+} U_{i}$. Thus

$$
M_{u}=\sum_{i=1}^{\mathrm{g}} k_{i} \bar{\beta}_{i}^{+} U_{i} p_{i}^{+}
$$

which implies that

$$
\begin{aligned}
E\left(M_{u}\right) & =E\left[\sum_{i=1}^{\mathrm{g}} k_{i} \bar{\beta}_{i}^{+} U_{i} p_{i}^{+}\right] \\
& =\sum_{i=1}^{\mathrm{g}} k_{i} \alpha_{s i}\left(\Pi_{I i}^{*}-p_{i} \Pi_{I i}^{+}\right)
\end{aligned}
$$

Theorem 4.4 : Let $I$ be the expected total number of incorrect decisions in a step-wise group screening design with $g$ group-factors in the initial step such that the $i^{\text {th }}$ group-factor of size $k_{i}$ contains factors with a-priori probability $\mathrm{p}_{\mathrm{i}}$ of being defective $(i=1,2, \ldots . ., g)$. Then

$$
I=\sum_{i=1}^{\mathrm{g}} k p-\sum_{i=1}^{\mathrm{g}} k_{i} p_{i} \Pi_{I i}^{+} \gamma_{s i}+\sum_{i=1}^{\mathrm{g}} k_{i} \alpha_{s i}\left(\Pi_{I i}^{*}-p_{i} \Pi_{I i}^{+}\right)
$$

Proof : The expected total number of incorrect decisions is given by

$$
\begin{aligned}
I & =I_{I}+I_{s}+E\left(M_{u}\right) \\
& =\sum_{i=1}^{\mathrm{g}} k_{i} p_{i}\left(1-\Pi_{I i}^{+}\right)+\sum_{i=1}^{\mathrm{g}} k_{i} p_{i} \Pi_{I i}^{+}\left(1-\gamma_{s i}\right)+\sum_{i=1}^{\mathrm{g}} k_{i} \alpha_{s i}\left(\Pi_{I i}^{*}-p_{i} \Pi_{I i}^{+}\right) \\
& =\sum_{i=1}^{\mathrm{g}} k_{i} p_{i}-\sum_{i=1}^{\mathrm{g}} k_{i} p_{i} \Pi_{I i}^{+} \gamma_{s i}+\sum_{i=1}^{\mathrm{g}} k_{i} \alpha_{s i}\left(\Pi_{I i}^{*}-p_{i} \Pi_{I i}^{+}\right)
\end{aligned}
$$

using (4.7), (4.8) and (4.9). This completes the proof.

\section{Corollary 4.1:}




$$
\begin{aligned}
\operatorname{Max} I & =\sum_{i=1}^{\mathrm{g}} k_{i} p_{i}-\sum_{i=1}^{\mathrm{g}} k_{i} p_{i} \gamma_{s i} \Pi_{I i}\left(\phi_{I i}, \alpha_{I i}\right) \\
& +\sum_{i=1}^{\mathrm{g}} k_{i} \alpha_{s i}\left\{\left(\alpha_{I i} q_{i}^{k_{i}}+\left(1-q_{i}^{k_{i}}\right) \Pi_{I i}\left(k_{i} \phi_{I i}, \alpha_{I i}\right)\right)-p_{i} \Pi_{I i}\left(\phi_{I i}, \alpha_{I i}\right)\right\}
\end{aligned}
$$

Proof:

$$
\begin{aligned}
I & =\sum_{i=1}^{\mathrm{g}} k_{i} p_{i}-\sum_{i=1}^{\mathrm{g}} k_{i} p_{i} \Pi_{I i}^{+} \gamma_{s i}+\sum_{i=1}^{\mathrm{g}} k_{i} \alpha_{s i}\left(\Pi_{I i}^{*}-p_{i} \Pi_{I i}^{+}\right) \\
& =\sum_{i=1}^{\mathrm{g}} k_{i} p_{i}-E\left(M_{R}\right)+E\left(M_{u}\right)
\end{aligned}
$$

Hence $I$ will take its maximum value when $E\left(M_{R}\right)$ is minimum and $E\left(M_{u}\right)$ is maximum. But $E\left(M_{R}\right)$ takes its maximum value when $\Pi_{I i i}^{+}$is replaced by $\Pi_{I i}\left(\phi_{I i}, \alpha_{I i}\right)$. That is

$$
\operatorname{Min} E\left(M_{R}\right)=\sum_{i=1}^{\mathrm{g}} k_{i} \gamma_{s i} p_{i} \Pi_{I i}\left(\phi_{I i}, \alpha_{I i}\right)
$$

$E\left(M_{u}\right)$ will take a minimum value when $\Pi_{I i i}^{*}$ is replaced by its maximum value and $\Pi_{I i}^{+}$is replaced by its minimum value. That is when $\Pi_{I i}^{*}$ is replaced by $\left\{\alpha_{I i} q_{i}^{k_{i}}+\left(1-q_{i}^{k_{i}}\right) \Pi_{I i}\left(k_{i} \phi_{I i}, \alpha_{I i}\right)\right\}$ and $\Pi_{I i}^{+}$is replaced by $\Pi_{I i}\left(\phi_{I i}, \alpha_{I i}\right)$.

Thus

$$
\operatorname{Max} E\left(M_{u}\right)=\sum_{i=1}^{\mathrm{g}} k_{i} \alpha_{s i}\left\{\alpha_{I i} q_{i}^{k_{i}}+\left(1-q_{i}^{k_{i}}\right) \Pi_{I i}\left(k_{i} \phi_{I i}, \alpha_{I i}\right)-p_{i} \Pi_{I i}\left(\phi_{I i}, \alpha_{I i}\right)\right\}
$$

The result follows on using (4.12) and (4.13) in (4.11).

Corollary 4.2 : For large $\frac{\Delta_{i}}{\sigma}$ and small $p_{i}{ }^{\prime} s$

$$
\operatorname{Max} \quad I \approx \sum_{i=1}^{\mathrm{g}} k_{i} \alpha_{s i}\left[\alpha_{I i}-p_{i}+\left(1-\alpha_{I i}\right) p_{i} k_{i}\right]
$$

Proof: For large $\frac{\Delta_{i}}{\sigma}$ and small $p_{i}{ }^{\prime} s, \Pi_{I i}\left(s_{i} \phi_{I i}, \alpha_{I i}\right) \approx 1, \gamma_{s i} \approx 1$ and $q_{i}^{k_{i}} \approx 1-k_{i} p_{i}$.

The result follows immediately on replacing these values by their approximations in the expression for Max I given in corollary 4.1.

\section{Optimum sizes of initial group-factors in relation to total cost}

We define the expected total $\operatorname{cost}(C)$ as a linear function of the expected number of runs and the expected number of incorrect decisions and obtain the sizes of the group-factors so that the expected total cost is minimum.

Let $c_{1}$ be the cost of inspection per run and $c_{2}$ be the loss for each incorrect decision made. Then the expected total cost is given by

$$
C=c_{1} E(R)+c_{2} \quad \operatorname{Max} I
$$




\section{M.M. MANENE}

We shall only discuss the case when $\frac{\Delta_{i}{ }^{\prime} s}{\sigma}$ are large and $p_{i}{ }^{\prime} s$ are small. For simplicity we shall only consider the special case when $\alpha_{I i}=\alpha_{I}, \alpha_{s i}=\alpha_{s}$ and $\alpha_{i}^{*}=\alpha^{*}(i=1,2, \ldots, g)$.

Theorem 5.1: If $\alpha_{I i}=\alpha_{I}, \alpha_{s i}=\alpha_{s}$ and $\alpha_{I}^{*}=\alpha^{*}$, then for large $\frac{\Delta_{i}{ }^{\prime} s}{\sigma}$ and small $p_{i}{ }^{\prime} s$, the value of $k_{i}$ which minimizes the expected total cost is given by

$$
k_{i}=\left(f-\sum_{i=1}^{g} G_{i} H_{i}\right) G_{i} / \sum_{i=1}^{g} G_{i}+G_{i} H_{i}
$$

where

$$
G_{i}=\frac{-1}{\left\{2\left(1-\alpha_{I}\right)-\left(1-\alpha_{s}\right)\left(1+\alpha^{*}\right)\right\} c_{1} p_{i}+2 \alpha_{s}\left(1-\alpha_{I}\right) c_{2} p_{i}}
$$

and

$$
H_{i}=c_{1}\left\{\alpha_{I}+\frac{1}{2}\left(1-\alpha_{s}\right)\left(3-\alpha^{*}\right) p_{i}\right\}-c_{2} \alpha_{s}\left(\alpha_{I}-p_{i}\right) ;(i=1,2, \ldots, \mathrm{g})
$$

Proof: The problem is to minimize

$$
C=c_{1} E(R)+c_{2} \quad \operatorname{Max} I
$$

subject to
(i) $\sum_{i=1}^{\mathrm{g}} k_{i}=f$
(ii) $k_{i}>0 \quad ; \quad i=1,2, \ldots, g$

That is to minimize

$$
\begin{aligned}
C= & c_{1}\left\{h+g+\alpha_{I} f-\left(1-\alpha_{s}\right) \sum_{i=1}^{\mathrm{g}} p_{i}+\left(1-\alpha_{I}\right) \sum_{i=1}^{\mathrm{g}} k_{i}^{2} p_{i}\right. \\
& \left.+\frac{1}{2}\left(1-\alpha_{s}\right)\left(3-\alpha^{*}\right) \sum_{i=1}^{\mathrm{g}} k_{i} p_{i}-\frac{1}{2}\left(1-\alpha_{s}^{*}\right) \sum_{i=1}^{\mathrm{g}} k_{i}^{2} p_{i}\right\} \\
& +c_{2}\left\{\alpha_{s} \alpha_{I} f-\alpha_{s} \sum_{i=1}^{\mathrm{g}} k_{i} p_{i}+\alpha_{s}\left(1-\alpha_{I}\right) \sum_{i=1}^{\mathrm{g}} k_{i}^{2} p_{i}\right\}
\end{aligned}
$$

subject to

(i) $\sum_{i=1}^{\mathrm{g}} k_{i}=f$

(ii) $k_{i}>0 \quad ; \quad i=1,2, \ldots, g$

using corollaries (3.1) and (4.2) noting that $\xi_{\mathrm{i}}=1, \alpha_{I i}=\alpha_{I}, \alpha_{s i}=\alpha_{s}$ and $\alpha_{i}^{*}=\alpha^{*}$.

Using the method of Lagrange multipliers, let 


\section{STEP-WISE GROUP SCREENIN DESIGNS}

$$
F\left(k_{1}, k_{2}, \ldots . ., k_{\mathrm{g}}, \lambda\right)=c_{1} E(R)+c_{2} \operatorname{Max} I+\lambda\left(\sum_{i=1}^{\mathrm{g}} k_{i}-f\right)
$$

where $\lambda$ is the Lagrange multiplier. Assuming continuous variation in $k_{i}$, the critical value of $k_{i}$ is obtained from the equations

$$
\partial F / \partial k_{i}=0 \quad ; \quad i=1,2, \ldots, g, \text { and } \partial F / \partial \lambda=0
$$

The theorem follows immediately on solving equations (5.3).

\section{Examples of screening plans}

The screening efficiency of step-wise group screening design with unequal group sizes can be measured in terms of the minimum expected total cost. A small value of $(C)$ indicates better performance on the average. Examples of group screening plans which minimize the expected total cost $(C)$ are given in Table 1 below. The corresponding values of $E(R)$ and Max $I$ are also given.

Table 1 : Optimum group-sizes obtained by minimizing expected total cost $(C)$, when $\alpha_{I i}=\alpha_{I}, \alpha_{i}^{*}=\alpha^{*}$ and $\alpha_{s i}=\alpha_{s}$, for $f=100$ and for selected unequal apriori probabilities. The minimum $(C)$ given is a relative figure using $c_{1}$ (the cost of observing a run) as the unit.

\begin{tabular}{|c|c|c|}
\hline$i$ & $p_{I}$ & $k_{I}$ \\
\hline 1 & 0.008 & 17.088 \\
\hline 2 & 0.009 & 15.024 \\
\hline 3 & 0.010 & 13.372 \\
\hline 4 & 0.013 & 9.942 \\
\hline 5 & 0.015 & 8.418 \\
\hline 6 & 0.017 & 7.252 \\
\hline 7 & 0.020 & 5.940 \\
\hline 8 & 0.022 & 5.265 \\
\hline 9 & 0.025 & 4.454 \\
\hline 10 & 0.027 & 4.014 \\
\hline 11 & 0.030 & 3.463 \\
\hline 12 & 0.033 & 3.013 \\
\hline 13 & 0.035 & 2.755 \\
\hline Total & & 100.000 \\
\hline
\end{tabular}

(a) $h=3, g=13, \alpha_{I}=\alpha^{*}=\alpha_{s}=0.05, \quad c_{2}: c_{1}=3: 5, \quad p_{i} \leq p=0.035$.

$E(R)=28.577$, Max $I=0.770$, Min $C=29.039$. The corresponding value of $\min$ $E(R)=26.739$ when incorrect observations are not considered.

(b) $h=4, g=20, \alpha_{I}=\alpha^{*}=\alpha_{s}=0.05, \quad c_{2}: c_{1}=3: 5, \quad p_{i} \leq p=0.100$. 


\section{M.M. MANENE}

\begin{tabular}{|c|c|c|}
\hline$i$ & $p_{I}$ & $k_{I}$ \\
\hline 1 & 0.040 & 9.565 \\
\hline 2 & 0.045 & 8.337 \\
\hline 3 & 0.050 & 7.354 \\
\hline 4 & 0.053 & 6.853 \\
\hline 5 & 0.055 & 6.550 \\
\hline 6 & 0.060 & 5.880 \\
\hline 7 & 0.062 & 5.642 \\
\hline 8 & 0.065 & 5.313 \\
\hline 9 & 0.070 & 4.827 \\
\hline 10 & 0.075 & 4.403 \\
\hline 11 & 0.078 & 4.179 \\
\hline 12 & 0.080 & 4.037 \\
\hline 13 & 0.082 & 3.902 \\
\hline 14 & 0.085 & 3.712 \\
\hline 15 & 0.087 & 3.592 \\
\hline 16 & 0.090 & 3.423 \\
\hline 17 & 0.092 & 3.316 \\
\hline 18 & 0.095 & 3.164 \\
\hline 19 & 0.098 & 3.020 \\
\hline 20 & 0.100 & 2.931 \\
\hline Total & & 100.000 \\
\hline
\end{tabular}

$E(R)=51.422, \operatorname{Max} I=1.545$, Min $C=52.349$. The corresponding value of $\min$ $E(R)=49.219$ when incorrect observations are not considered.

From the two tables it is easily seen that when a cost function involving both $E(R)$ and Max $I$ is used, the number of runs increases. It should be noted that these tables are just an illustration. The values of $p_{i}$ 's used are not unique; neither is the ratio $c_{2}: c_{1}$.

\section{References}

DORFMAN, R. 1943. The delection of defective members of large populations, Annals of Mathematical Statistics, 14: 438 -440.

LI, C.H. 1962. A sequential method for screening experimental variables. Jour. Arner Statistical Assoc. 57: 455-477.

MANENE, M.M. 1985. Further investigations of group screening designs: Step-wise designs; $\mathrm{Ph} . \mathrm{D}$. thesis submitted to University of Nairobi.

MANENE, M.M. 1997. On Two Type Step-wise group screening designs, Proc of fifth scientific con£. Of Eastern. Central and Southern Africa Network of I. B.S., $57-62$.

ODHIAMBO, J.W. and MANENE, M.M. 1987. Step-wise Group Screening Designs with Errors in Observations. Comm. In Statistics Theor. Meth. 16(1 0): 3095-3115.

ODHIAMBO, J.W. and PATEL, M.S. 1986. On Multistage Group Screening Designs. Comm. in Statistics Theor. Meth. 15(5): 1627-1645.

OTTIENO, J.A.M. and PATEL M.S. 1984. Two Stage Group Screening Designs with Unequal Apriori Probabilities. Comm in Statistics Theor. Meth. 13(6): 761-779.

PATEL, M.S. 1962. Group Screening with more than Two Stages; Technometrics, 4: 209-217. 


\section{STEP-WISE GROUP SCREENIN DESIGNS}

PATEL, M.S. and MANENE, M.M. 1987. Step-Wise Group Screening with Equal Prior Probabilities and no errors in Observations, Comm. In Statistics Simula_and Comguta, 16(3): 817-833.

PLACKET, R.L. and BURMAN, J.P 1946. The Design of Optimum Multifactor Experiments. Biometrika, 33: 305-325.

STERRET, A. 1957. On the Detection of Defective Members of Large Populations, Annals of Mathematical Statistics. 28: 1033-1036.

WATSON, G.S. 1961. A study of the Group Screening Method, Technometrics, 3: 371-388.

Received 30 December 2002

Accepted 20 November 2003 